\title{
Mutualists construct the ecological conditions that trigger the transition from parasitism
}

\author{
Léo Ledru ${ }^{1}$, Jimmy Garnier ${ }^{2}$, Matthias Rohr ${ }^{1}$, Camille Noûs $^{3}$, Sébastien Ibanez ${ }^{1}$ \\ ${ }^{1}$ Univ. Grenoble Alpes, Univ. Savoie Mont Blanc, CNRS, LECA, 38000 Grenoble, France \\ ${ }^{2}$ CNRS, Univ. Grenoble Alpes, Univ. Savoie Mont Blanc, LAMA, 73000 Chambery, France \\ ${ }^{3}$ Laboratory Cogitamus
}

Address for correspondence: leo.ledru@univ-smb.fr

\begin{abstract}
The evolution of mutualism between hosts and initially parasitic symbionts represents a major transition in evolution. Although vertical transmission of symbionts during host reproduction and partner control both favour the stability of mutualism, these mechanisms require specifically evolved features that may be absent during the transition. Therefore, the first steps of the transition from parasitism to mutualism are not fully understood. Spatial structure might be the key to this transition. We explore this hypothesis using a spatially explicit agent-based model. We demonstrate that, starting from a parasitic system with global dispersal, the coevolution between mutualistic effort and local dispersal of hosts and symbionts leads to a stable coexistence between parasites and mutualists. The local dispersal evolution mimics vertical transmission and triggers the formation of mutualistic clusters, counteracting the individual selection level of parasites that maintain global dispersal. However, the transition also requires competition between hosts in order to occur. Indeed, the transition occurs when mutualistic symbionts increase the density of hosts, which strengthens competition between hosts and disfavours parasitic host/symbiont pairs: mutualists create ecological conditions that allow their own spread. Therefore, the transition to mutualism may come from an eco-evolutionary feedback loop involving spatially structured population dynamics.
\end{abstract}

Keywords: mutualism, major transition, spatial structure, dispersal evolution, eco-evolutionary feedbacks, niche construction

\section{Introduction}

In their bestseller, Szathmáry and Smith [1995] explored several major transitions in biology, from the origin of chromosomes to the evolution of social groups. Most of these transitions resulted from the formation of a larger entity from smaller entities. In addition, smaller entities specialize within the larger ones and often become dependent on them (e.g., for reproduction), eventually making the transition irreversible.

Following the work of Queller [1997], Szathmáry [2015] adopted a dichotomy between fraternal transitions such as multicellularity arising from a division of labour among closely related units and egalitarian transitions such as mutualistic symbiosis [Bronstein, 2015], where more distant units come together to complement their functions in a larger unit.

For a transition to occur and persist, evolutionary conflicts between the subentities must not overtake the whole's fate. In the case of fraternal transitions, this is prevented by the strong relatedness between subentities [Hamilton, 1964a,b, Queller, 2000, Fisher et al., 2013]. However, in the case of egalitarian transitions, the subentities generally belong to different species. Thus, it can be advantageous for them to remain autonomous and exploit the other subentities. This selfish behaviour occurs at the expense of the whole, as for the tragedy of the commons [Garrett, 1968, Hardin, 1998]. For instance, a parasitic symbiont may remain parasitic rather than collaborate in mutualistic symbiosis [Drew et al., 2021]. The resulting evolutionary conflict might be circumvented by vertical transmission of the symbionts, which ensures that all subentities share a common fate [Wilson and Sober, 1994]. As a result, vertical transmission of symbionts promotes the transition to mutualism [Maynard, 1998, Herre et al., 1999, Wilkinson and Sherratt, 2001, Ferdy and Godelle, 2005, Kerr and Nahum, 2011, Akçay, 2015, Estrela et al., 2016, Queller and Strassmann, 2016].

The importance of vertical transmission has been highlighted by experiments on microbial systems [Sachs et al., 2011, Shapiro et al., 2016, King et al., 2016, Shapiro and Turner, 2018] as well as in natura observations of a

Author contributions: SI, JG and LL originally formulated the project; all authors developed the model; LL and MR performed the numerical analyses; LL, SI and JG wrote the manuscript. 
Wolbachia-insect system [Weeks et al., 2007].

However, in many mutualistic systems, the symbiont is transmitted horizontally [Wilkinson and Sherratt, 2001], such as legume-rhizobium [Denison and Kiers, 2004], squid-vibrio [McFall-Ngai, 2014], mycorrhizae [Allen, 1991] or plant ants [Bronstein et al., 2006, Rico-Gray and Oliveira, 2008]. In such cases, several mechanisms such as partner selection, sanction or fidelity and costly exploitation, can counteract the selection for selfishness [GenkaiKato and Yamamura, 1999, Wilkinson and Sherratt, 2001, Sachs et al., 2004, Foster and Wenseleers, 2006, Estrela et al., 2016, Akçay, 2017].

For instance, in legume-rhizobium, mycorrhizal and plant-ant associations, the plants can sanction the less beneficial (or even detrimental) symbionts by allocating them fewer resources [West et al., 2002, Kiers et al., 2003, Denison and Kiers, 2004, Edwards et al., 2006, Bever et al., 2009, Akçay, 2015].

However, it is unclear whether these mechanisms are present at the beginning of the transition. Since they require the evolution of complex and specific traits, they may occur in later stages, providing additional stability to the system. In the absence of such traits, what mechanism could promote the start of a transition to mutualism? Using a theoretical model, the present work aim to show that the coevolution between mutualistic effort and local dispersal of hosts and symbionts triggers the formation of mutualistic clusters which can resist the invasion of parasites. In addition to the spatial structure, we show that the competition between hosts truly promotes the start of the transition.

A similar issue exists with respect to the evolution of altruism, since partner choice/control mechanisms, such as voluntary reciprocal altruism [Axelrod, 1981], may be restricted to higher animals or may appear during later evolutionary stages. Spatial structure has been recognized as a general mechanism promoting the transition to altruism [Mitteldorf and Wilson, 2000, Lion and Baalen, 2007, 2008, Débarre et al., 2012]. Spatial structure generally stems from local dispersal, which triggers the formation of clusters dominated by altruistic individuals, while individuals with similar phenotypes are positively assorted in space [Wilson and Dugatkin, 1997, Pepper, 2007]. The balance between individual-level selection favouring cheaters and cluster-level selection favouring altruists ultimately determines the evolutionary outcome [Baalen and Rand, 1998, Mitteldorf and Wilson, 2000]. Moreover, the coevolution of cooperation and dispersal can allow the emergence of altruism, with spatial clusters of altruistic individuals promoting the persistence and spread of altruistic phenotypes [Koella, 2000, Le Galliard et al., 2005, Hochberg et al., 2008, Purcell et al., 2012, Mullon et al., 2018]. Empirical evidence on the evolution of reduced virulence [Boots and Mealor, 2007], the evolution of altruism [Harcombe, 2010], and the evolution of restraint predation [Kerr et al., 2006] also supports the crucial role of the spatial structure.

With respect to altruism, spatial structure can allow mutualists to overcome non-mutualists [Yamamura et al., 2004, Akçay, 2017], and this can involve the evolution of dispersal [Mack, 2012]. However, this may not be sufficient to account for the transition from parasitism to mutualism, since parasitic symbionts should discourage hosts from initiating the transition, whereas non-mutualists have a weaker impact [Yamamura et al., 2004, Mack, 2012]. Moreover, in previous attempts, mutualistic efforts were initially polymorphic but were not subject to mutations [Mack, 2012]. In that case, mutualistic clusters cannot be invaded from inside through parasitic mutations, which favours mutualism. The present work therefore constitutes, to our knowledge, the first spatially explicit eco-evolutionary model where the mutualistic efforts and dispersal abilities of hosts and symbionts coevolve, beginning from a fully parasitic interaction. If some hosts and symbionts simultaneously become mutualists and start dispersing locally, this may lead to the formation of mutualistic host/symbiont clusters producing more offspring than parasitic host/symbiont pairs, thereby initiating the transition. Meanwhile, parasitic symbionts should continue dispersing globally to invade such clusters, which could homogenize the spatial structure and compromise the transition. Therefore, it is unclear whether mutualists will invade, whether mutualists will replace parasites, or whether both strategies will coexist, as is often the case in nature (e.g., [Després and Jaeger, 1999, Borges, 2015]).

The concept of major transitions also implies that the host and the symbiont become dependent upon each other [Szathmáry and Smith, 1995, Szathmáry, 2015], with each partner needing the other to perform essential functions. Dependence is often accompanied by gene loss and gene exchange, rendering the transition irreversible [Estrela et al., 2016]. For instance, aphids and their intracellular bacterial symbiont Buchnera aphidicola are irreversibly mutually dependent since the symbiont provides essential amino acids to its host [Akman Gündüz and Douglas, 2009, Bennett and Moran, 2015]. However, most mutualistic interactions do not exhibit such physiological or reproductive dependencies (e.g., plant-ants or legume-rhizobium mutualisms), and several reverse pathways are often possible from mutualism to parasitism [Sachs and Simms, 2006, Werner et al., 2018, Week and Nuismer, 2021]. Since the present work focuses on eco-evolutionary dynamics, we will not consider any physiological or developmental dependency or irreversibility and will instead assume that hosts are always able to produce offspring when alone. Nevertheless, the number of offspring produced by the hosts can depend on the mutualistic efforts of both species as well as on the population densities, which are expected to change during the transition. Under these altered ecological conditions, isolated hosts may exhibit a negative population growth rate, although they are physiologically able to produce offspring. This is hereafter called "ecological dependence".

To sum up, we tackle the following issues: 
- Main question: Can the transition from parasitism to mutualism occur when mutualistic effort coevolves with local dispersal in the absence of vertical transmission and partner control?

- Q1: How do mutualistic clusters arise during the first steps of the transition? Is their formation necessary for the transition?

- Q2: By maintaining global dispersal, can non-mutualistic hosts and parasitic symbionts coexist with mutualists?

- Q3: Do mutualistic hosts become ecologically dependent on their symbiont?

To investigate these questions, we built an agent-based model using a two-dimensional space lattice that supports an autonomous host and a host-dependent symbiont. Hosts compete for space and other resources, while symbionts compete for available hosts. This situation occurs in many biological systems, such as plant-ant, plant-fungi, or plant-seed eating pollinator. To model the transition from parasitism, the symbiont is initially detrimental to the host, and the host provides it the minimal energy possible, without any spontaneous mutualistic effort, as would be the case after an antagonistic evolutionary arms race. Moreover, the host-parasite system is ecologically viable even in the absence of any mutualistic agent in the landscape. At first, both species disperse globally; this situation corresponds to the most disadvantageous conditions for the emergence of mutualism. Through continuous mutations, mutualistic and locally dispersing symbionts and hosts can appear, establish and eventually change the population dynamics, triggering feedback on their own evolutionary dynamics. In addition to these general hypotheses, no assumptions specific to a particular biological system were required.

\section{Model description}

Main rules Our model considers two types of agents, hosts and symbionts, living on the same two-dimensional space lattice. The interaction between the two species occurs when they share the same cell. Each cell can assume three states: i) empty, ii) occupied by a solitary host (only one host is allowed per cell), iii) occupied by a hostsymbiont couple, (only one symbiont is allowed per host). Each individual brings two traits, the mutualistic interaction trait $(\alpha)$ and the dispersal trait $(\varepsilon)$. Both influence fecundity.

At every time point, agents undergo each following steps (see appendix A.1 and Figure A1 for more details and schematic representation of the life cycle):

- The host and symbiont die with fixed probability $m$.

- They produce offspring, possibly with different traits from them due to mutations. The fecundity of the parents depends on their traits and on their interactions with their possibly cell-sharing partner.

- The offspring are dispersed according to the parents' dispersal traits $\varepsilon$.

- The host offspring may establish only in empty cells, while the symbiont offspring can only establish in cells already occupied by a solitary host. If several individuals come to implant in the same cell, a uniform lottery determines which one will implant, while the others die.

Fecundity rate and mutualism/parasitism The fecundity rate $f$ of an agent depends on its mutualistic interaction trait $\alpha$ as well as the trait of its cell-sharing partner. This continuous trait, ranging between 0 and 1 , determines the intensity of the agent investment in the mutualistic relationship. We assume a positive interaction trait dependence between agents. A mutualistic agent tends to increase the fecundity of its cell-sharing partner. On the other hand, a mutualistic agent has an intrinsic cost, reducing its fecundity. Thus, the effective agent fecundity results from the gain induced by the mutualistic behaviour of its partner weighted by its own intrinsic cost of mutualism (for mathematical details see appendix A.1).

In addition, the cost of mutualism is a developmental characteristic that applies regardless of the actual interaction. Thus, a solitary mutualistic host always pays the cost of mutualism. Therefore, we consider "strong mutualism", where no direct feedback occurs for mutualists and mutualism always has a cost.

The hosts are autonomous, i.e., their fecundity is nonzero when they are alone in their cell without a symbiont. In addition, while hosts are always beneficial to symbionts, because they are necessary for their establishment, symbionts can be parasitic to hosts. Indeed, we assume that the presence of a symbiont induces a cost for the host. Thus, if the symbiont invests insufficiently in the mutualistic interaction, it does not compensate for this cost, and the host has reduced fecundity than if it was alone; the symbiont is parasitic to the host. The symbiont becomes mutualistic when it sufficiently invests in the mutualistic interaction so that the host has a higher fecundity than if it was alone. In our model, a symbiont is parasitic if $\alpha<0.475$ and mutualistic if $\alpha>0.475$ (see Figure 1).

We note interaction traits of the host and the symbiont $\alpha_{h}$ and $\alpha_{s}$, respectively. The fecundity rate ranges between a positive low value that depends on the agent type and a maximum rate of 8 corresponding to the maximum number of direct neighbours in a $2 \mathrm{D}$ space (Figure 1). 

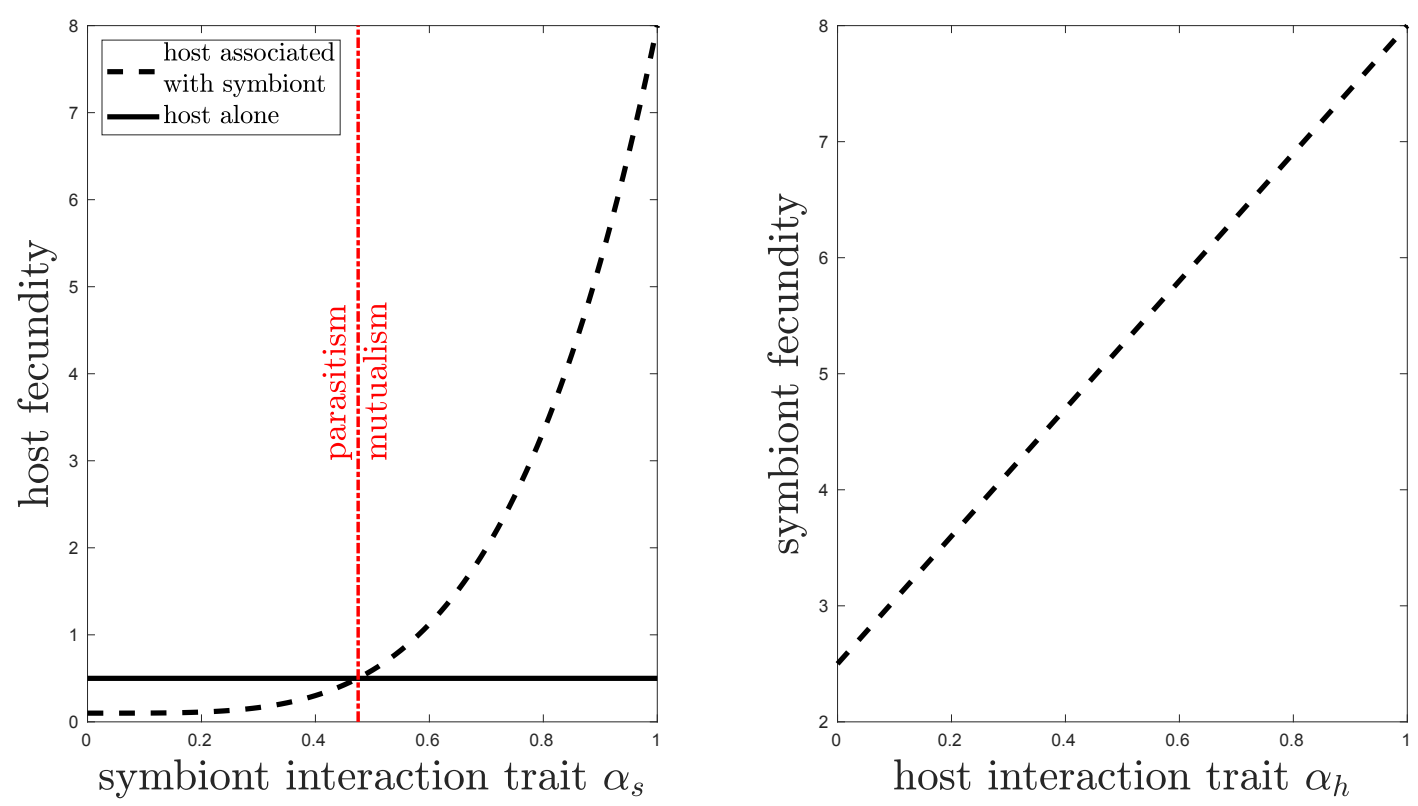

Figure 1: Fecundity rates of hosts and symbionts according to the interaction trait of their partners (dashed lines). Plain line: fecundity of a solitary host. The dashed-dotted red line corresponds to the threshold $\alpha_{s}^{*}$ between the parasitic symbiont (solitary host fecundity higher than the fecundity of a host associated with a symbiont of trait $\alpha_{s}<\alpha_{s}^{*}$ ) and mutualistic symbiont (solitary host fecundity lower than fecundity of a host associated with a symbiont of trait $\alpha_{s}<\alpha_{s}^{*}$ ).

Mutation Offspring inherit traits from their parents with variability due to mutations. The effects of mutations on each trait are independent, and can be either beneficial or detrimental. We describe these effects using an exponential distribution that allows for rare mutations with large effects. We set a maximum mutation distance $d$; if a distance from the exponential distribution is greater than $d$, the mutation is cancelled. Similarly, a mutation is cancelled if it implies that the trait exceeds the limits of its domain $[0,1]$.

Dispersal The parents are sessile, whereas their descendants are dispersed. The individuals can invest in two modes of dispersal, a local dispersal considering the 8 neighbouring cells of the parent's cell and a global dispersal corresponding to a uniform dispersal across the entire space.

Their dispersal trait $\varepsilon$ provides the proportion of offspring globally dispersed uniformly in the space. The remaining proportion $1-\varepsilon$ is locally dispersed uniformly in the cells around the parent. Similar dispersal was used in Kéfi et al. [2007, 2008].

Global dispersal implies an additional cost that reduces the number of offspring. Therefore, effective fecundity $f_{e}$ is equal to the fecundity rate $f$ reduced by a cost that is linearly proportional to the investment in global dispersal: $f_{e}=f(1-d \varepsilon)$, with $d$ a coefficient to modulate cost intensity.

Competition The host is subject to intraspecific density-dependent competition, which influences the establishment success of the offspring. The establishment probability $P_{I}$ decrease with the host density $\rho_{h}$ as follows:

$$
P_{I}=1-\left(\rho_{h}\right)^{\gamma_{C}}
$$

where $\rho_{h}$ is the proportion of cells occupied by hosts and $\gamma_{C}$ is the competition strength. The competition is strong when $\gamma_{C}<1$ (sub-linear function), while it is weak when $\gamma_{C} \geq 1$ (super-linear function).

Parasitic system and transition To tackle the issue of transition to mutualism, we assume that the system is viable without mutualism. More precisely, we chose parameters such that the extinction probability of a population with parasitic global dispersers (minimal interaction traits, $\alpha_{s}=\alpha_{h}=0$ and dispersal trait $\varepsilon=1$ ) was less than 1 in the absence of mutation. Moreover, this parasitic population without evolution stabilizes around a demographic equilibrium called the "parasitic system". From our perspective, this situation is the worst-case scenario because interactions are parasitic and dispersal cost is maximal. However, under evolution, mutations generate diversity, and mutualistic symbionts can appear. Starting from the parasitic system, mutations lead to the presence of approximately $2 \%$ of mutualistic symbionts in the population. They are not selected but simply 
result from the phenotypic variability induced by mutations. Then, the system can either remain in this state, or a transition to mutualism can occur. The transition is characterized by a significant increase of the percentage of mutualistic symbionts above the percentage generated by simple phenotypic variability. In our following work, we assume that transition occurs when the percentage of mutualistic symbionts rises above the threshold of $10 \%$.

\section{Results}

In the following results, the maximum cost of mutualism is $30 \%$, and the other parameters are set to satisfy the viability of the parasitic system (see Table A1 in appendix A.1).

The transition from parasitism to mutualism under restrictive conditions Our first objective was to investigate whether in the absence of dispersal cost, the transition to mutualism is possible starting from the viable parasitic system without dispersal cost. It represents the most difficult condition for the transition to mutualism. From our simulations, we can compute the transition time when the percentage of mutualistic symbionts rises above $10 \%$, which is our transition threshold.

Without dispersal cost, the transition is more likely to occur under strong competition than weak competition (see the probability of transition in Figure 2a)). Moreover, when the transition succeeds, it occurs more rapidly under strong competition (median transition time around $7.10^{4}$ ) than under weak competition (median transition time around $\left.2.5 \cdot 10^{4}\right)$. However, with a large dispersal cost $(d=0.45)$, the transition occurs systematically (the probability of transition is 1) and quickly (the median transition time is divided by a hundred $7.10^{2}$ ), regardless of whether the competition is weak or strong (see Figure $2 \mathrm{a}$ ).

The transition is characterised not only by an increase in mutualistic symbionts but also by an increase in host and parasitic symbiont densities (see Figure $2 \mathrm{~b}$ and $\mathrm{c}$ ). During the transition, we also observe an increase in the mean interaction trait of mutualistic symbionts toward 1 (see curve colour in Figure 2c). Thus, the transition is initiated by weakly mutualistic symbionts, which then rapidly increase their mutualistic interaction. In contrast, the increase in the average host interaction trait is delayed in response to the symbionts' transition (see curve colour in Figure 2b). Moreover, the transition does not occur at the expense of parasitic symbionts. The latter benefits from the increase in host density created by the mutualistic symbionts. As a result, their density increases so that the parasitic symbionts remain dominant (Figure 2c).

Note that if the cost of mutualism is sufficiently low (e.g., a maximum cost of only $10 \%$ instead of the $30 \%$ of the previous results), the transition to mutualism occurs even in the absence of host competition (details not shown).

The evolutionary branching resulting from the transition is accompanied by coevolution with the dispersal trait (see Figure 3a). As a result, we obtain two classes of symbionts: parasitic global dispersers $\left(\alpha_{s}<<1\right.$ and $\varepsilon \sim 1)$ and mutualistic local dispersers $\left(\alpha_{s} \sim 1\right.$ and $\left.\varepsilon<<1\right)$. Conversely, the host trait distribution is more aggregated, and coevolution with the dispersal trait leads to a negative correlation between global dispersal and mutualism intensity (Figure $3 \mathrm{~b}, \mathrm{R}^{2}=0.102$, $\mathrm{p}$-value=0). Moreover, we observe that very few hosts exhibit a weak interaction trait $\left(\alpha_{h}<0.2\right)$. The low density of very weak mutualistic hosts results from the apparent competition between hosts due to a high density of symbionts. In this situation, a host is frequently associated with a symbiont. Strong mutualistic hosts will benefit more from parasitic symbionts than weak mutualistic hosts. Indeed, a parasitic symbiont will benefit from a strong mutualistic host by increasing its number of offspring, which eventually reduces the host density and thus host competition.

To investigate whether the transition to mutualism is associated with the emergence of a spatial structure, we measured an index of assortment (see appendix A.1 for details). A positive index indicates that on average, individuals with similar phenotypes are closer than individuals with different phenotypes when compared to a random spatial distribution of the individuals in the space (Figure 4a). The intraspecific assortment quantifies the similarity of agents in a local neighbourhood ( 8 cells), and the interspecific assortment quantifies the similarity between host and symbiont in the same cell.

Positive assortment indices demonstrate that the agents are locally similar and that hosts and symbionts in the same location also have the same interaction behaviour. As a result, we show that the transition to mutualism occurs along with the emergence of a spatial structure (see clusters of hosts and mutualistic symbionts Figure 4b and c). The intraspecific spatial structure is stronger than the interspecific structure. This is not surprising since the formation of the intraspecific spatial structure simply requires that a sufficient proportion of the dispersal is local. However, for an interspecific spatial structure to emerge, a phenotype must have a sufficiently local dispersal, and it must interact with a similar phenotype that also exhibits a local dispersal.

Effect of competition We have shown that competition between hosts promotes the transition to mutualism. We next investigated its quantitative effect on the percentage of mutualistic symbionts. The following results were obtained using a large dispersal cost $(d=0.45)$ to reduce the mean time of transition and thus save computational time. We also discuss the effect of dispersal cost in the next section.

Competition strength increases the percentage of mutualistic symbionts after the transition when competition is global over the landscape, i.e., hosts compete with all the hosts in the landscape (Figure 5a). However, when 

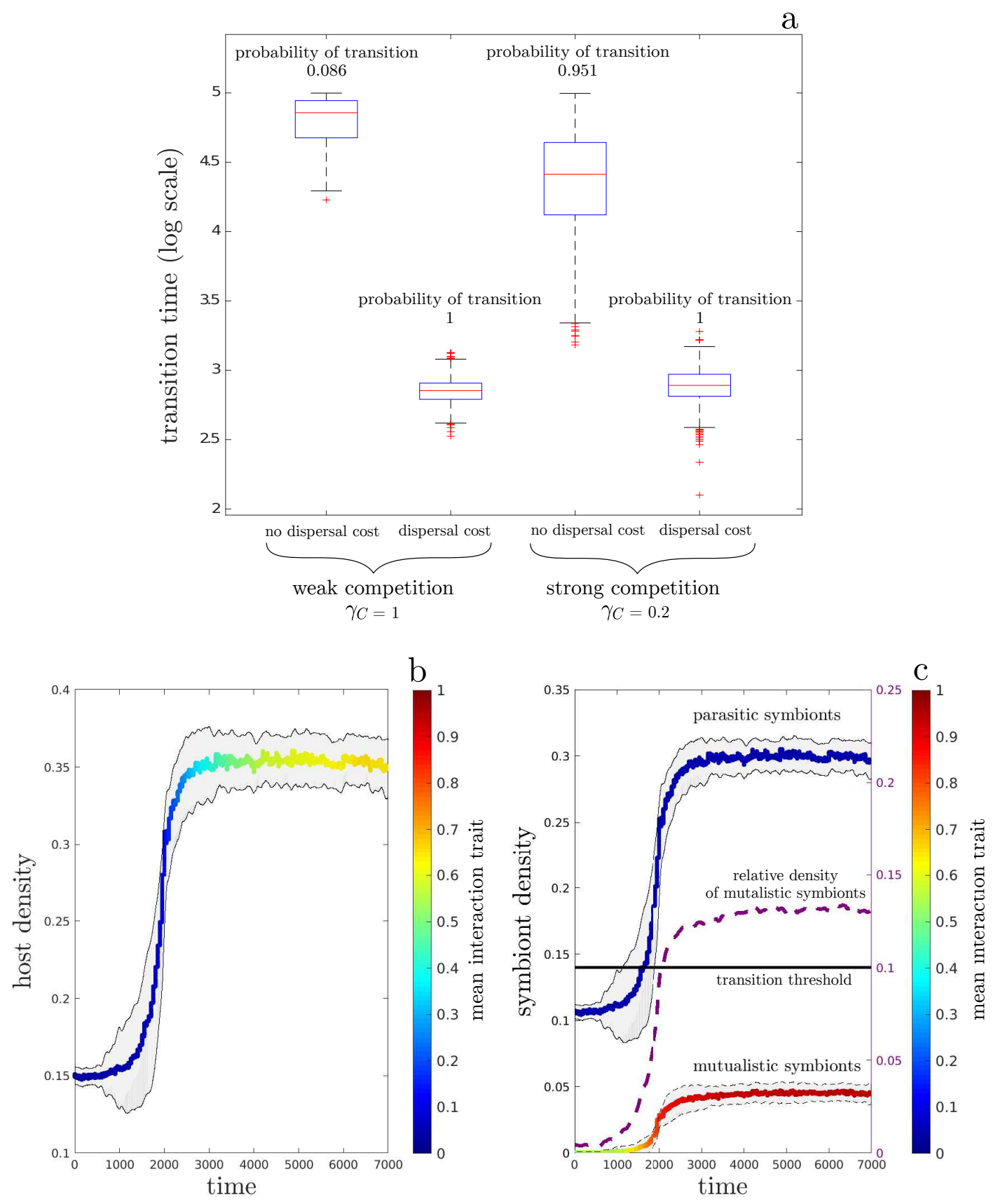

Figure 2: a) Transition time as a function of competition strength $\gamma_{C}$ and dispersal cost $d$. Boxplots representing the transition time when the percentage of mutualistic symbionts increases above $10 \%$ when starting from the parasitic system. We ran 1000 replicates per parameter pair with a maximum projection time of $10^{5}$. The probability of transition corresponds to the frequency of transition over the 1000 simulations. In panels b) and c), coloured plain curves represent the host and symbiont densities over time averaged over 100 simulations under strong competition $\gamma_{C}=0.2$ and no dispersal cost $d=0$. The time series are adjusted so that all simulations have a transition time $t=2000$. The colour gradient corresponds to the mean interaction trait $\alpha$, and shaded regions correspond to the standard deviation for densities. In panel c), the purple dotted line and the right y-axis show the relative density of mutualistic symbionts, and the black line indicates the $10 \%$ transition threshold.

competition is more local, individuals compete only with their neighbours (see appendix A.1 and Figure A2 for details), and the percentage of mutualistic symbionts decreases drastically (Figure 5b). When the competition is too local, the average percentage of mutualistic symbionts eventually drops below the transition threshold, and no transition occurs.

Finally, we showed that mutualism persists only because of strong competition. Indeed, if we reduce the 
bioRxiv preprint doi: https://doi.org/10.1101/2021.08.18.456759; this version posted August 19, 2021. The copyright holder for this preprint (which was not certified by peer review) is the author/funder, who has granted bioRxiv a license to display the preprint in perpetuity. It is made available under aCC-BY-NC-ND 4.0 International license.
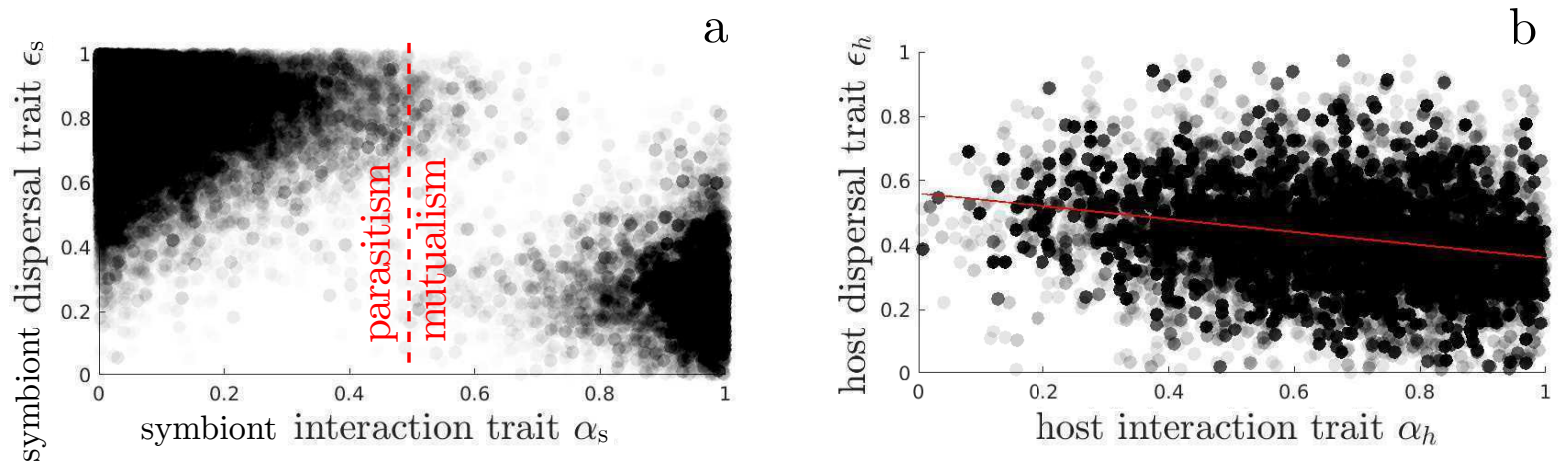

Figure 3: Distribution after transition to mutualism in traits domain $(\varepsilon, \alpha)$ of symbionts (panel a) and hosts (panel b). Plain red line (panel b) shows the linear regression between host traits $\left(\mathrm{R}^{2}=0.102\right.$, p-value $=0)$. Distributions corresponds to 100 simulations with strong competition $\gamma_{C}=0.2$ and no dispersal cost $d=0$.

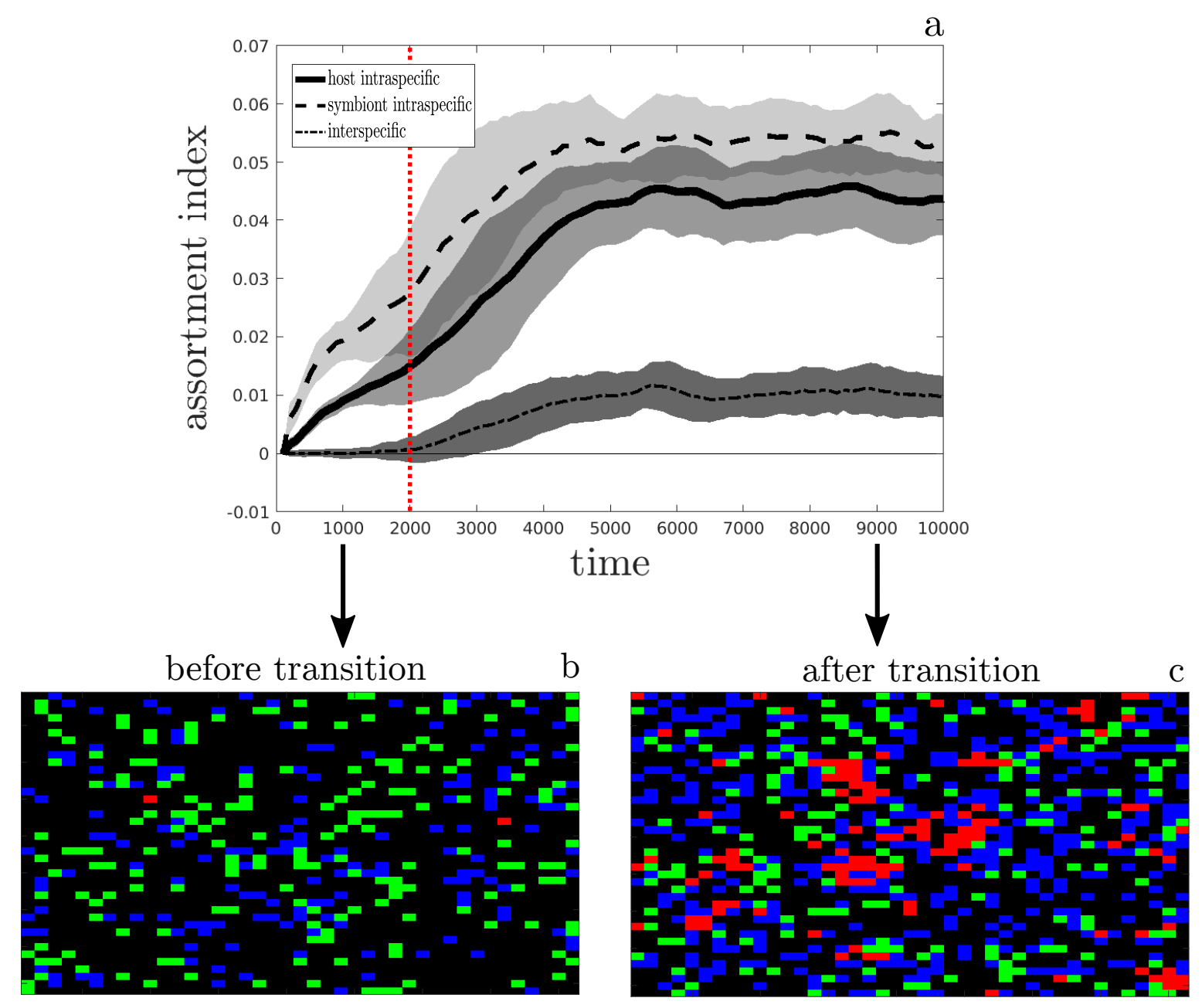

Figure 4: a) Spatial structures are described by the assortment index measuring the intraspecific assortment between hosts (plain line), the intraspecific assortment between symbionts (dashed line) and the interspecific assortment between hosts and symbionts (dash-dot line). Results are averaged over 100 simulations with strong competition $\gamma_{C}=0.2$ and no dispersal cost $d=0$. The time series are adjusted so that all simulations have a transition time $t=2000$ (red dotted line). Grey areas show the standard deviation. b)-c) Snapshots of a region of 40x40 cells before (panel b) and after (panel c) the transition to mutualism. Coloured cells represent host alone (green), host with parasitic symbiont (blue), host with mutualistic symbiont (red), empty (black). 

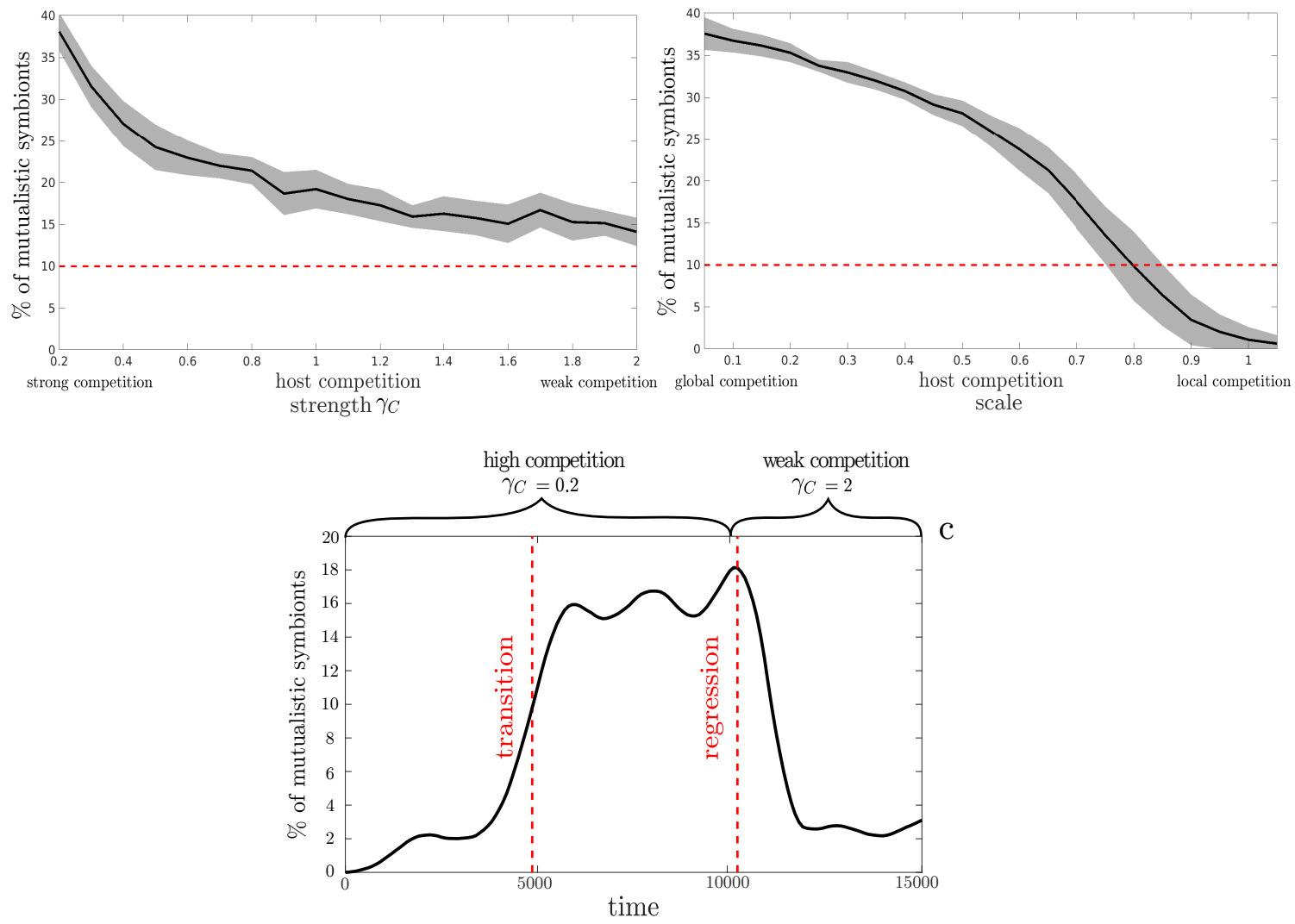

Figure 5: The role of host competition in the transition to mutualism. a) The effect of the competition intensity (defined by the competition shape parameter $\gamma_{C}$ ) on the transition to mutualism (global competition). b) The effect of the spatial scale of the competition (strong competition $\gamma_{C}=0.2$ ). c) The effect of competition on the transition and on the maintenance of mutualism (simulation without dispersal cost). Panels a and b show the results averaged over 50 simulations with a maximum projection time of $10^{4}$ steps, and grey areas give the standard deviation. The red dotted line is the threshold of $10 \%$ of mutualistic symbionts above which transition to mutualism occurs.

competition after a transition to mutualism, we observe that the population evolves towards the parasitic system (see Figure $5 \mathrm{c}$ and Figure A3 in the appendix for more details).

Another way to reduce density-dependent competition is to reduce the density of the host. In this case, we show that mutualism still persists (Figure A4a in appendix). Thus, temporal perturbations in host density tend to enhance the proportion of mutualism. Indeed, the perturbation itself does not change the proportion of mutualistic symbionts because the different symbionts are fairly evenly distributed throughout the space. Then, the space vacated by the perturbation is recolonised mostly by parasitic symbionts due to their more global dispersal (Figure A4b). However, due to the relaxation of global competition, the probability of host establishment is better, and the mutualistic clusters outside the perturbation zone gain in size, which explains why the proportion of mutualistic symbionts increases slightly despite the recolonization of the centre by parasites. In the end, once recolonization is complete, the system returns to an equilibrium state close to before the perturbation. In terms of trait distribution, the new state is similar to the previous state before the perturbation (details not shown). The same experiment but with a perturbation that causes the death of $50 \%$ of uniformly occupied cells leads to the same results.

Mortality and dispersal cost can induce host dependency in emerging mutualistic systems. Competition is a major process leading to mutualism. However, mortality and dispersal cost also play important roles in shaping the mutualistic system. Thus, for the results below, the competition is both strong and global.

We demonstrated that dispersal cost and mortality promote mutualism (Figure 6). Although the mutualistic system emerges in a wide range of parameters where the parasitic system is viable, the transition is more likely to occur when the system is close to extinction. We demonstrated that the transition is less likely to occur when the dispersal cost is low. In particular, for the parameter pair in the area indicated by the grey star, we have shown previously that the probability of transition is very low around 0.086 (see Figure 2). In addition, we show 


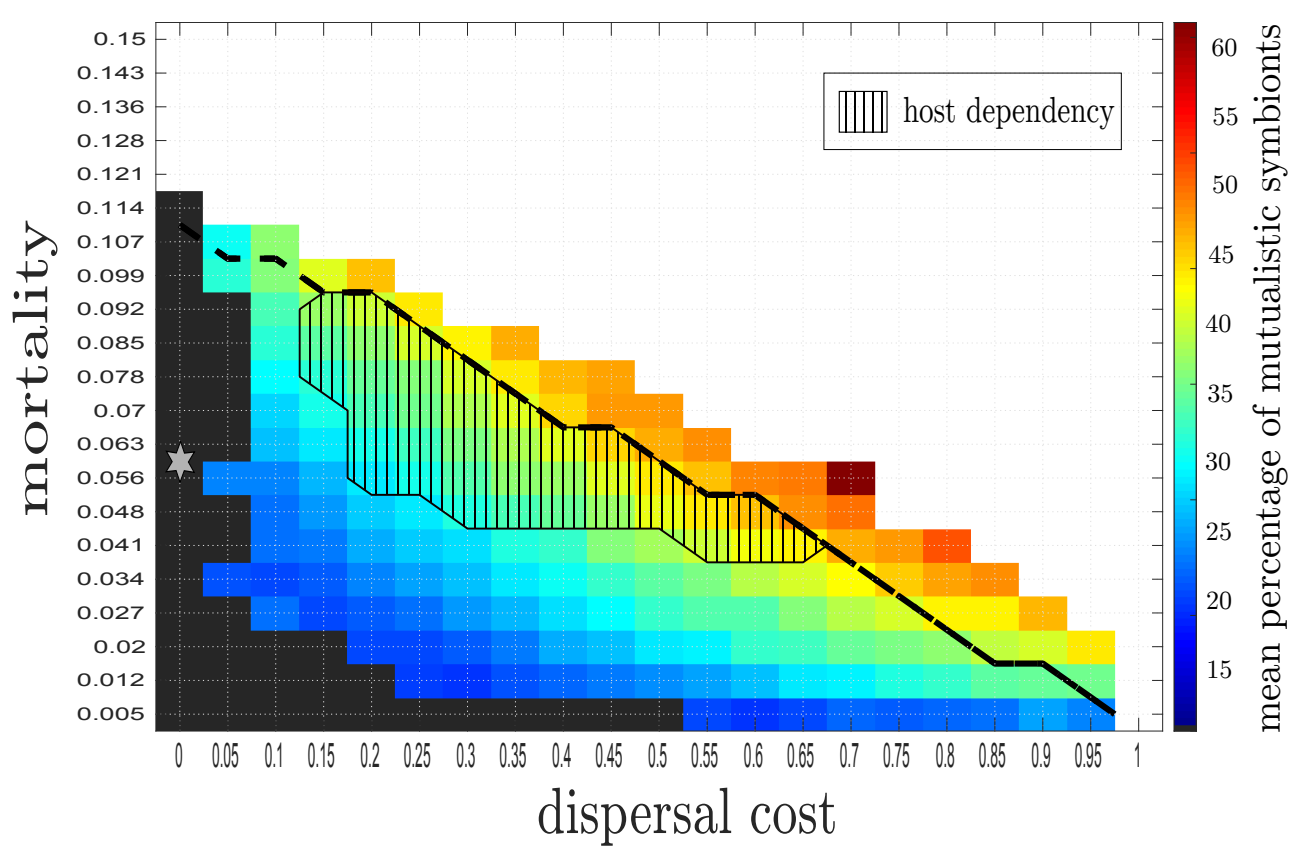

Figure 6: The percentage of mutualistic symbionts when there is transition to mutualism as a function of intrinsic mortality and dispersal cost. We performed 50 simulations per parameter with strong global competition $\gamma_{C}=0.2$. The percentages are averaged over the simulations that lead to transition, if any occur. The white cells correspond to the nonviability domain for the system with evolution, and above the black dotted line, the parasitic system is not viable. The dark grey area corresponds to no transition for all simulations. The evolution of host ecological dependency occurs in the hatched area. Ecological dependency means that the average host from a population where mutualism has emerged cannot survive the competition experienced by that population (i.e. the ecological conditions) if the mutualistic symbionts are removed. The grey star corresponds to the restrictive conditions of Figure 3; note that under these conditions, a transition is possible but rare (probability of transition is 0.086 ), so with only 50 simulations per parameter no transition occurs here.

that the transition is unlikely to occur if the mortality is too low, which tends to demonstrate that the time of emergence increases if the mortality decreases.

The dispersal cost also modifies the characteristics of the system by reducing the average dispersal trait of both species (Figure A5 and Table A2 in the appendix). This reduction is associated with more pronounced branching of the symbiont due to the increase in the percentage of mutualistic symbionts. We observed that even in response to a strong dispersal cost, the mean dispersal trait of parasitic symbionts is higher than the mean dispersal trait of mutualistic symbionts.

Under favourable conditions leading to the transition to mutualism, the population of mutualistic hosts always persists in the absence of the population of mutualistic symbionts. Therefore, in our model, the transition to mutualism does not exhibit an absolute dependency between host and symbiont populations. However, ecological dependency may occur. The transition to mutualism cooccurs with an increase in host density and thus an increase in intraspecific competition. If this increase in competition is sustainable only in the presence of mutualistic symbionts, we say that the hosts are ecologically dependent on the symbionts. To test this dependence, we measured the global competition between hosts in a population at equilibrium after a transition to mutualism. Then, we used this competition as a fixed non-density-dependent competition to test whether a host population with all individuals having the same trait as the mean trait of a population at equilibrium can survive in the absence of mutualistic symbionts and mutation. We show that this dependency occurs when the system evolves toward a mutualistic system in which the percentage of mutualistic symbionts is sufficiently large (Figure 6 hatched area).

\section{Discussion}

The mechanism underlying the transition to mutualism. In relation to our primary question, our results indicate that the transition from parasitism to mutualism occurs when mutualistic efforts coevolve with dispersal, despite the absence of vertical transmission or partner control. Before the transition, the host performs 
better when alone; therefore, it has no interest in increasing its mutualistic effort; in fact, it is selected to keep it as low as possible. In contrast, the symbiont population is limited by the number of available hosts, which increases when the symbiont becomes mutualistic. If by chance mutations produce a mutualistic symbiont dispersing locally and interacting with a host dispersing locally as well, its offspring will benefit from the increased density of hosts in their neighbourhood and will form a mutualistic cluster (question Q1, Figure 4c). The cluster will be rapidly invaded by parasitic symbionts dispersing globally, which can lead to its extinction if competition between hosts is weak. Under such conditions, the transition to mutualism rarely occurs (Figure 2a), and when it does, the percentage of mutualistic symbionts remains low (Figure 5a). Conversely, when hosts strongly compete for resources, the ecological conditions change dramatically. The formation of mutualistic clusters (Figure 4a) increases population densities (Figures $2 \mathrm{~b}$ and $2 \mathrm{c}$ ), which enhances competition between hosts. Areas dominated by parasitic host/symbiont pairs were initially viable, but their population growth rate became negative following the increase in competition. This creates empty space that can be colonized by mutualists, which still disperse globally from time to time. Therefore, contrary to our expectation, the transition is not directly caused by the higher fecundity of mutualistic clusters but only indirectly by the increase in host competition. However, this only occurs when competition between hosts is partly global (Figure 5b); if it is purely local, mutualistic clusters cannot influence the viability of parasitic regions. Although areas dominated by parasitic host/symbiont pairs become unsuitable, parasitic symbionts can still invade mutualistic clusters and overcome them. This results in a dynamic equilibrium between mutualism and parasitism (question Q2, Figure 2c). Coevolution with dispersal results in a negative correlation between mutualism intensity and global dispersal ( $80 \%$ of mutualists disperses locally, Figure 3), which mirrors the link between altruistic behaviour and local dispersal [Koella, 2000, Le Galliard et al., 2005, Hochberg et al., 2008, Purcell et al., 2012, Mullon et al., 2018]. Empirical work has shown that the outcome of interactions between hosts and symbionts depends not only on the traits of the protagonists, but also on the ecological conditions [Bronstein, 1994]. For instance, plants take advantage of seed-eating pollinators in the absence of alternative pollinators but not in their presence [Thompson and Cunningham, 2002]. Mycorrhizae are beneficial for plants when soil resources are scarce while they are detrimental when resources are abundant [Johnson et al., 1997]. In the above cases, the outcome of the interaction depends on both biotic and abiotic factors that are external to the host-symbiont system. Our model showed that the association with symbionts remains parasitic when host competition is low, while it evolves towards mutualism when host competition increases. In that case, the outcome of the interaction depends on intrinsic features of the interactions that are constructed by the eco-evolutionary dynamics of the system, as the emergence of mutualists increases host density.

The transition needs some time to occur because several obstacles must be overcome (simultaneity of the mutations, demographic stochasticity, susceptibility to invasions by parasites) before the mutualists are numerous enough to induce the shift in host competition. For this reason, the dispersal cost was introduced to speed up the transition (Figure 2a) without affecting the overall behaviour of the model. Dispersal cost induces a selection pressure at the individual level in favour of local dispersal, which increases the likelihood of the formation of mutualistic clusters.

In addition to dispersal cost, mortality enhances the probability of transition. Mortality pushes the parasitic system towards its viability boundary and promotes the eco-evolutionary feedback loop involving host competition. The facilitation of mutualistic symbiosis in harsh environmental conditions has already been observed theoretically and empirically [Callaway et al., 2002, Maestre et al., 2003, Travis et al., 2006]. However, explaining mutualistic behaviour it is not always a significant process [Werner et al., 2015]. Moreover, in the context of altruism, an inverse relationship is observed [Taylor and Irwin, 2000].

The role of quasi-vertical transmission Although mutualistic symbionts are environmentally acquired, their local dispersal, together with mutualistic hosts, produces a similar effect as vertical transmission (as for mycorrhizae, [Wilkinson, 1997]), which we term "quasi-vertical" transmission. However, local dispersal (even $100 \%$ ) is not equivalent to vertical transmission because host and symbiont offspring can disperse to any of the 8 neighbouring cells. Vertical transmission due to specific reproductive and physiological adaptations would have produced transitions to mutualism more easily. Moreover, the colonization of empty space by a mutualistic pair requires the simultaneous long-distance dispersal of both species, whereas in the case of vertical transmission, this always occurs. In addition, the need to colonize empty space constitutes an important selection pressure, which partly counteracts the necessity of quasi-vertical transmission. Indeed, mutualistic phenotypes retain their ability to disperse globally ( $\sim 20 \%$ for symbionts and $\sim 40 \%$ for hosts, Figure 3$)$, which cannot be due to mutations alone. Colonization ability comes along with the avoidance of local overpopulation generated by mutualism. Similarly, although the evolution of altruism is favoured by kin selection (itself induced by local dispersal [Hamilton, 1964b]), it can also be limited by kin competition [Wilson et al., 1992, Alizon and Taylor, 2008, Akdeniz and van Veelen, 2020].

A mixed strategy combining both dispersal modes takes advantage of kin selection and simultaneously maintains the opportunity to escape kin competition. Figure $5 \mathrm{~b}$ shows that purely local competition between hosts prevents the transition to mutualism because kin competition overcomes kin selection in that case with respect to the evolution of cooperation [Akdeniz and van Veelen, 2020]. In nature, global competition between hosts may 
arise when plants compete for water present in the same groundwater, while competition for light is more local. Thus, the evolution of mutualism may depend on the dominant form of competition for resources between hosts.

The interplay between three levels of organization The mechanism by which parasitic symbionts and hosts can invade mutualistic clusters is a two-species version of the tragedy of the commons [Garrett, 1968, Feeny et al., 1990, Hardin, 1998], which can be bypassed by local dispersal in the case of altruism [Mitteldorf and Wilson, 2000] as well as in the present case. Indeed, there is no transition to mutualism when dispersal is primarily global $(\varepsilon>0.55)$, mutualists and parasites coexist for mixed dispersal strategy $(0.55>\varepsilon>0.1)$, and mutualists almost outcompete parasites when dispersal is mainly local $(\varepsilon<0.1$, details not shown). In the context of altruism, the resolution of the tragedy of the commons involves two levels of organization, the individual-level favouring cheaters and the group-level favoring altruism [Baalen and Rand, 1998]. In the context of mutualism, parasites are selected at the individual level and mutualists at the host/symbiont pair level, resembling the tit-for-tat strategy where cooperators are selected at the pair level. However, this is not the end of the story because in the absence of dispersal cost, mutualism rarely invades when host competition is weak (Figure 2a), despite the occasional formation of mutualistic pairs. For the transition to occur, competition between hosts at the global scale is necessary (Figure 5b), which constitutes a third level of organization involved in the coevolutionary process.

Host dependency and irreversibility of the transition Major transitions in evolution are characterized by the irreversibility and the interdependence between the agents [Szathmáry and Smith, 1995, Estrela et al., 2016]. Our model does not include any physiological dependence of the host on its symbiont or any loss of functions in the host due to gene transfers, assuming that this generally occurs during later stages of the evolution of mutualism. Instead, we have defined dependence from a population dynamics perspective: the host is "ecologically dependent" when its population growth rate is negative in the absence of its symbiont, even though it can produce offspring (question Q3). We found that mutualistic hosts deprived of their symbiont exhibit a negative growth rate when the host density after the transition to mutualism becomes sufficiently large (Figure 6). In our model, this ecological dependency resulted from the density-dependent competition between hosts and the assumption that mutualism is costly for the host, even when its symbiont is absent (e.g., the unconditional production of extrafloral nectaries [Bronstein, 1998]). However, this dependency is not absolute. Indeed, once the density becomes sufficiently low, the mutualistic hosts alone are viable. The dependency may become absolute for a sufficiently high cost of mutualism, but in these conditions, the transition to mutualism will not occur.

If host competition strength decreases permanently, e.g., following the continuous supply of extra resources, the reverse transition back to parasitism occurs (Figure 5c). This reversion occurs because host dependency relies on the ecological conditions that change when the host competition strength is modified. However, if host competition decreases punctually, e.g., following a perturbation of a fraction of the landscape, mutualism persists (Figure A4) because mutualistic clusters take advantage of the reduction of global host competition to colonize free cells around them. This leads to an increase in host competition; in that case, mutualism can restore the ecological conditions, allowing its own persistence, as in a niche construction process [Lewontin, 1982, 1983, Odling-Smee et al., 2013, Laland et al., 2016]. Niche construction is generally understood as the improvement of abiotic conditions. In the context of mutualism, it is due to the improvement of host densities, which induces an increase in host competition. This also occurs at the beginning of the transition, when the first mutualistic clusters trigger an increase in global host density.

Conclusion In the present paper, we aim to understand the mechanisms promoting the transition from parasitism to mutualism. To tackle this issue, we develop an agent based model on a lattice. In our general model, we only assume that the mutualistic interactions influence the fecundity of both partners and that hosts face density-dependent competition. In addition, we ensure that the antagonistic system is stable in absence of mutations. We show that in the absence of vertical transmission or partner control mechanisms, the coevolution between mutualistic effort and local dispersal can trigger the transition from parasitism to mutualism, provided that intraspecific competition between host is sufficiently global and that either dispersal cost or competition strength is large enough.

Unexpectedly, we found that mutualistic clusters invade the antagonistic system thanks to their ability to increase the population densities of both partners, thereby triggering global competition between hosts and rendering regions dominated by parasitic host/symbiont pairs unsuitable. In contrast, the higher fecundity of mutualists is not advantageous enough to compensate for the ability of parasites to invade mutualistic clusters. Thus, our results suggest that the eco-evolutionary feedback involving competition between hosts might promote the transition from parasitism to mutualism in a wide range of biological systems, such as plant-fungi, plant-ant and plant-seed-eating pollinator interactions. 
Acknowledgements We are grateful to Jean-François Arnoldi and Cédric Gaucherel for their thoughtful comments and discussion on this work.

\section{A Appendix}

\section{A.1 Mathematical and numerical details of the model}

We present here the mathematical underpinnings of the model as well as some details of the numerical computation.

Algorithm Our model follows the cycle presented in Figure A1:

- Host and symbiont die with fixed probability $m \in(0,1)$.

- They produce offspring, possibly with different traits from them due to mutation. The fecundity of the parents depends on their two traits $(\alpha, \varepsilon) \in[0,1]^{2}$ and on their interactions with their possibly cell-sharing partner.

- The offspring are dispersed according to the parents' dispersal traits $\varepsilon$.

- The offspring of the hosts may establish only in empty cells, while the offspring of the symbionts can only establish in cells already occupied by a solitary host. If several individuals arrive in the same cell, a lottery determines which one will establish, while the others die.

In our numerical computations, mutations occurred only after the descendant was successfully established in a cell. This procedure saves computational time and did not influence our results because offspring dispersal and establishment do not depend on their traits but only on their parent traits. Furthermore, the mortality process was applied to both types of agents simultaneously, while the reproduction and dispersal processes were applied consecutively to the hosts and then to the symbionts. We confirmed that the order of the algorithm did not qualitatively affect our results.

Fecundity rate The fecundity rate $f$ of an agent depends on its mutualistic interaction trait $\alpha$ as well as the interaction trait of its cell-sharing partner. This continuous trait ranging between 0 and 1 determines the intensity of the agent investment in the mutualistic relationship.

We assumed a positive interaction trait dependence between agents. A mutualistic agent tends to increase the fecundity of its cell-sharing partner. The interaction fecundity $f_{I}^{i}\left(\alpha_{j}\right)$ of an individual of type $i \in\{h, s\}$, (h $=$ host, $\mathrm{s}=$ symbiont) interacting with an individual of type $j \in\{s, h\}$ with trait $\alpha_{j}$ was defined by

$$
\begin{aligned}
& f_{I}^{h}\left(\alpha_{s}\right)=f_{I, \min }^{h}\left(1+c_{f}^{h} f_{I, \min }^{h} \alpha_{s}^{\gamma_{f}}\right) \\
& f_{I}^{s}\left(\alpha_{h}\right)=f_{I, \min }^{s}\left(1+c_{f}^{s} f_{I, \min }^{s} \alpha_{h}\right) \\
& \text { with } c_{f}^{h}=\frac{f_{I, \max }-f_{I, \min }^{h}}{f_{I, \min }^{h}} \text { and } c_{f}^{s}=\frac{f_{I, \max }-f_{I, \min }^{s}}{f_{I, \min }^{s}}
\end{aligned}
$$

The coefficient $\gamma_{f}$ allows us to set the influence of trait $\alpha_{s}$ on fecundity. Using a coefficient $\gamma_{f}<1$, we create a convex function allowing a transition from parasitism to mutualism for a central value of the symbiont interaction trait. However, note that modifying the shape of this fecundity curve (from concave to convex via linear) does not qualitatively change our results.

On the other hand, a mutualistic agent has an intrinsic cost reducing its fecundity. The mutualism cost $c_{m}\left(\alpha_{i}\right)$ of an individual of type $i \in\{h, s\}$ ( $\mathrm{h}=$ host, $\mathrm{s}=$ symbiont) ranges between 0 and 1 , and it increases with interaction trait $\alpha_{i}$ of the agent. It is defined by

$$
c_{m}^{i}\left(\alpha_{i}\right)=1-c \alpha_{i}
$$

where $c$ is the maximal cost of mutualism.

Thus, for the host as for the symbiont, the fecundity $f_{i}$ of an individual $i$ interacting with an individual $j$ is the product of the interaction fecundity $f_{I}\left(\alpha_{j}\right)$ defined by (2) and the cost of mutualism $c_{m}\left(\alpha_{i}\right)$ defined by $(3)$.

$$
\begin{aligned}
f_{h} & =c_{m}^{h}\left(\alpha_{h}\right) f_{I}^{h}\left(\alpha_{s}\right) \\
f_{s} & =c_{m}^{s}\left(\alpha_{s}\right) f_{I}^{s}\left(\alpha_{h}\right)
\end{aligned}
$$

When a host agent is alone in a cell, its fecundity is defined by its intrinsic host fecundity $f_{a}^{\max }$ weighted by its mutualism cost $c_{m}\left(\alpha_{h}\right)$ : Fecundity of the solitary host:

$$
f_{a}=c_{m}^{h}\left(\alpha_{h}\right) f_{\max }^{a} .
$$




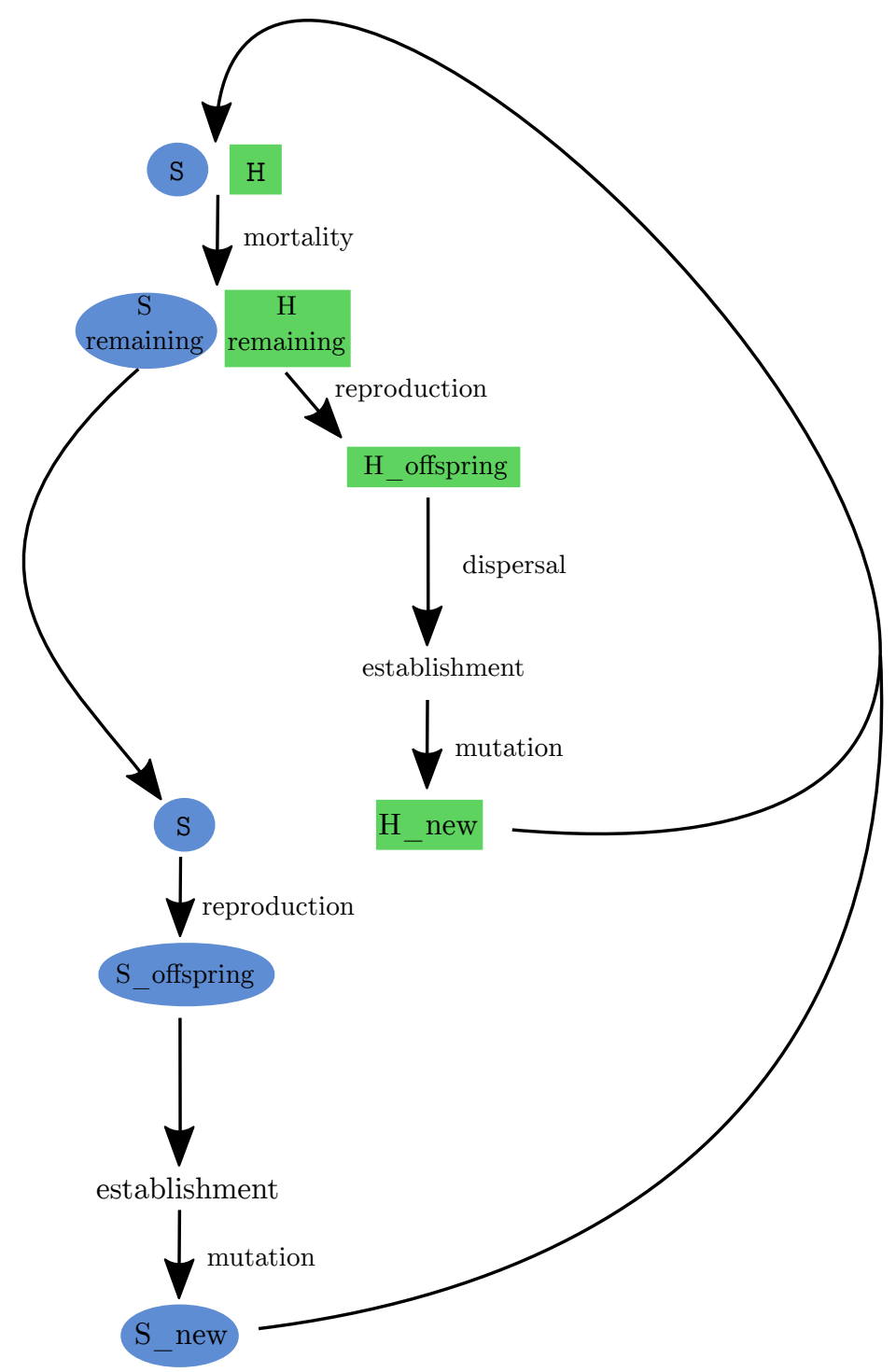

Figure A1: Diagram of the numerical algorithm. The host population $(\mathrm{H})$ and the symbiont population (S) undergo intrinsic mortality, then reproduction, dispersal, establishment, and finally mutation. The mortality step is simultaneous for the host and the symbiont, while the other steps occur first for the host and then for the symbiont.

Fecundity still depends on the interaction trait of the host but we replace the interaction fecundity $f_{I}^{h}\left(\alpha_{s}\right)$ with an intrinsic fecundity $f_{m a x}^{a}$. We therefore consider that the cost of mutualism is a developmental cost that is paid regardless of whether the interaction is realized. It can therefore be said that the mutualism modelled here is a "strong mutualism" in the sense that there is no direct return on investment for the partner with a mutualist effect, and the cost of mutualist behaviour is always paid.

The fecundity rates produced by our process were rarely integers, yet the number of offspring in our model can only be represented by an integer. Thus, in the numerical algorithm, the fecundity was rounded down to the lower integer, and then the decimal part is used as a parameter of a Bernouilli trial to determine whether a descendant was added.

Mutualism/parasitism threshold In our model, the presence of a host always produced a net benefit for the symbiont. However, the presence of the symbiont might be detrimental for the host. Indeed, the fecundity of a host $h$ interacting with a symbiont $s$ is $f_{I}\left(\alpha_{s}\right) c_{m}\left(\alpha_{h}\right)$, while the fecundity of the same host $h$ without a symbiont is $f_{a}^{\max } c_{m}\left(\alpha_{h}\right)$. Thus, the host has net benefit only if its fecundity in association with a symbiont is larger than its fecundity alone. Therefore, mutualism only occurs when $f_{I}\left(\alpha_{s}\right)>f_{a}^{\max }$. Otherwise, the interaction is parasitic. This criterion does not depend on the host mutualism trait $\alpha_{h}$ because the hosts always pay the same mutualism 
cost. Thus, we can define the mutualism/parasitism threshold $\alpha_{s}^{*}$ such that $f_{I}\left(\alpha_{s}^{*}\right)=f_{a}^{\text {max }}$; thus, we obtain

$$
\alpha_{s}^{*}=\left(\frac{f_{\text {max }}^{a}-f_{I, \min }^{h}}{c_{f}^{h} f_{I, \text { min }}^{h}}\right)^{1 / \gamma_{f}} \approx 0.475
$$

with the parameters set in Table A1.

Competition To test the effect of the spatial scale of the competition, we introduced a scale parameter $c_{s} \in$ $[0,1]$ that weighs the effect of local $\rho_{h}^{\text {local }}$ and global $\rho_{h}^{\text {global }}$ host density on the competition. The establishment probability thus satisfies

$$
P_{I}=1-\left(\left(1-c_{s}\right) \rho_{h}^{\text {local }}+c_{s} \rho_{h}^{\text {global }}\right)^{\gamma_{C}}
$$

The local host density $\rho_{h}^{\text {local }}$ corresponds to the host density in the 8 neighbouring cells surrounding the implantation cell of the host, while the global density $\rho_{h}^{\text {global }}$ corresponds to the host density all over the landscape (see Figure A2 for a schematic representation).

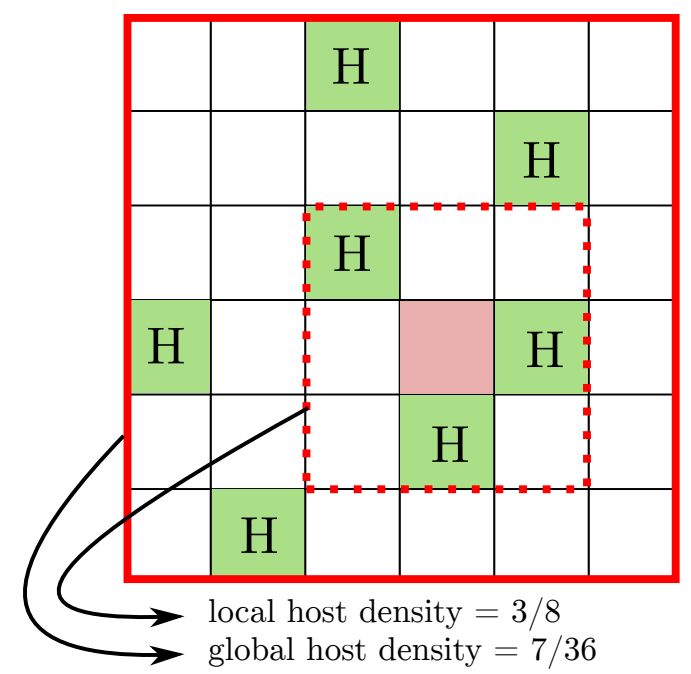

Figure A2: Local and global host densities influence the probability of establishment in the focus cell (pink filled square). The global density corresponds to the host density in the whole 36-cell landscape. The local density corresponds to the density in the eight cells vicinity around the focus cell.

These competition scales may have various ecological explanations. For instance, plants sharing the same water table face global competition for this resource. Conversely, the competition for light between plants is an example of local competition. Thus, our competition scale model allows us to describe the competition for several different resources that may appear at different scales. Following our previous examples, if the water supply represents $90 \%$ of the competition and light supply represents only $10 \%$, then the competition scale $c_{s}$ is $c_{s}=0.9$ (90\% global competition and $10 \%$ local competition).

Assortment index To compute the assortment index, we measured the similarity between spatially neighbouring phenotypes for the space resulting from the transition to mutualism and for a space containing the same phenotypes but whose distribution in space was randomised. The assortment index corresponds to the difference between the measurement made on the space resulting from the transition to mutualism and the measurement on the randomly rearranged space. If the index shifts positively (resp. negatively) from zero, it means that similar phenotypes are closer (resp. more distant) than different phenotypes compared to random spatial distribution. This methodology is similar to that used in Pepper and Smuts [2002] and Pepper [2007].

In practice, for the intraspecific assortment index, we utilized the following algorithm for each species: i) for each occupied cell by the target species, we measured the difference between the interaction trait present in the cell and the average of the interaction traits present in the neighbouring cells (a maximum of 8 neighbours can be present), and then we averaged these measurements to obtain an average similarity $S$ at the scale of the whole space; ii) this operation was repeated on 1000 spaces containing the same interaction traits but randomly redistributed between the occupied cells to obtain the average similarity corresponding to a random spatial distribution $S_{r}$; iii) finally, the assortment index corresponds to $S-S_{r}$.

For the interspecific assortment index, the first step was different: i) for each cell occupied by a host-symbiont pair, we measured the difference between their interaction traits, and then we averaged all of these measurements to obtain an average similarity $S$. Steps ii and iii follow the same methods as described above. 
bioRxiv preprint doi: https://doi.org/10.1101/2021.08.18.456759; this version posted August 19, 2021. The copyright holder for this preprint (which was not certified by peer review) is the author/funder, who has granted bioRxiv a license to display the preprint in perpetuity. It is made available under aCC-BY-NC-ND 4.0 International license.

\begin{tabular}{cl}
\hline Parameters & Values \\
\hline mortality $m$ & 0.06 \\
mutualism cost $c$ & 0.3 \\
upper limit of host and symbiont interaction fecundity $f_{I, \text { max }}$ & 8 \\
lower limit of host interaction fecundity $f_{I, \min }^{h}$ & 0.1 \\
lower limit of symbiont interaction fecundity $f_{I, \text { min }}^{s}$ & 2.5 \\
upper limit of solitary host fecundity $f_{\text {max }}^{\text {sh }}$ & 0.5 \\
shape coefficient of the host interaction fecundity $\gamma_{f}$ & 4 \\
maximum mutation distance $d$ & 0.5 \\
\hline
\end{tabular}

Table A1: Fixed parameters used for results unless otherwise specified.

\section{A.2 Competition strength, perturbation and mutualism persistence}

Competition strength determines mutualism persistence In the main text, we show that competition is essential for the transition to mutualism, but it is also important for its persistence, as shown here. In this section, we explored the effect of a sudden variation in competition strength $\gamma_{C}$ on the persistence of mutualism. We started with a strong competition $\gamma_{C}=0.2$. As expected from our previous results, a transition to mutualism occurred (see Figure A3a), c) and d)). Then, around $t=10^{4}$, we suddenly switched the competition strength to $\gamma_{C}=2$, corresponding to weak competition. We observed regression of mutualism due to the proportion of mutualistic symbionts decreasing from $20 \%$ to less than $5 \%$ (see Figure A3a) and e)). We observed that the regression of mutualism due to a weakening of the competition corresponded with an increase in host and symbiont densities. This increase is due to the reduction of competition, which determines densities more than the presence or absence of mutualism does.
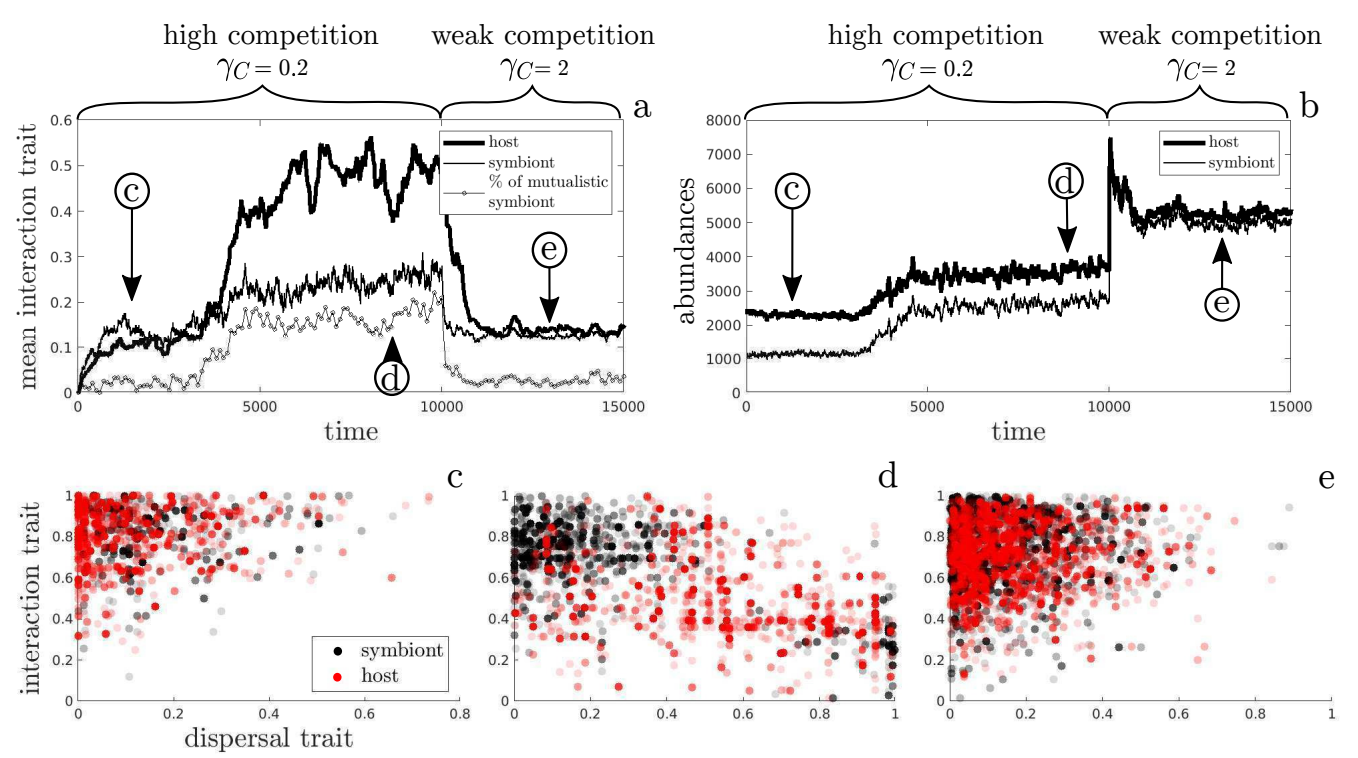

Figure A3: The transition to mutualism and collapse of mutualism depending on competition. a) Host and symbiont average interaction traits and the percentage of mutualistic symbionts over time. b) Host and symbiont abundance. There is high competition from time $=0$ to time $=10000$ and weak competition from time $=10001$ to the end. c) Dispersal and interaction traits domain before the transition to mutualism. d) Dispersal and interaction traits domain during mutualism persistence. e) Dispersal and interaction traits domain after mutualism collapse.

Density perturbation does not affect mutualism persistence Next, we tested how mutualism responds to a decrease in competition due to eradication of hosts and symbionts in a large homogeneous region of space (Figure A4). While previously we demonstrated that mutualism regresses when competition is set to be weak, we show here that mutualism persists in the face of decreased competition due to decreased host density. 
bioRxiv preprint doi: https://doi.org/10.1101/2021.08.18.456759; this version posted August 19, 2021. The copyright holder for this preprint (which was not certified by peer review) is the author/funder, who has granted bioRxiv a license to display the preprint in perpetuity. It is made available under aCC-BY-NC-ND 4.0 International license.
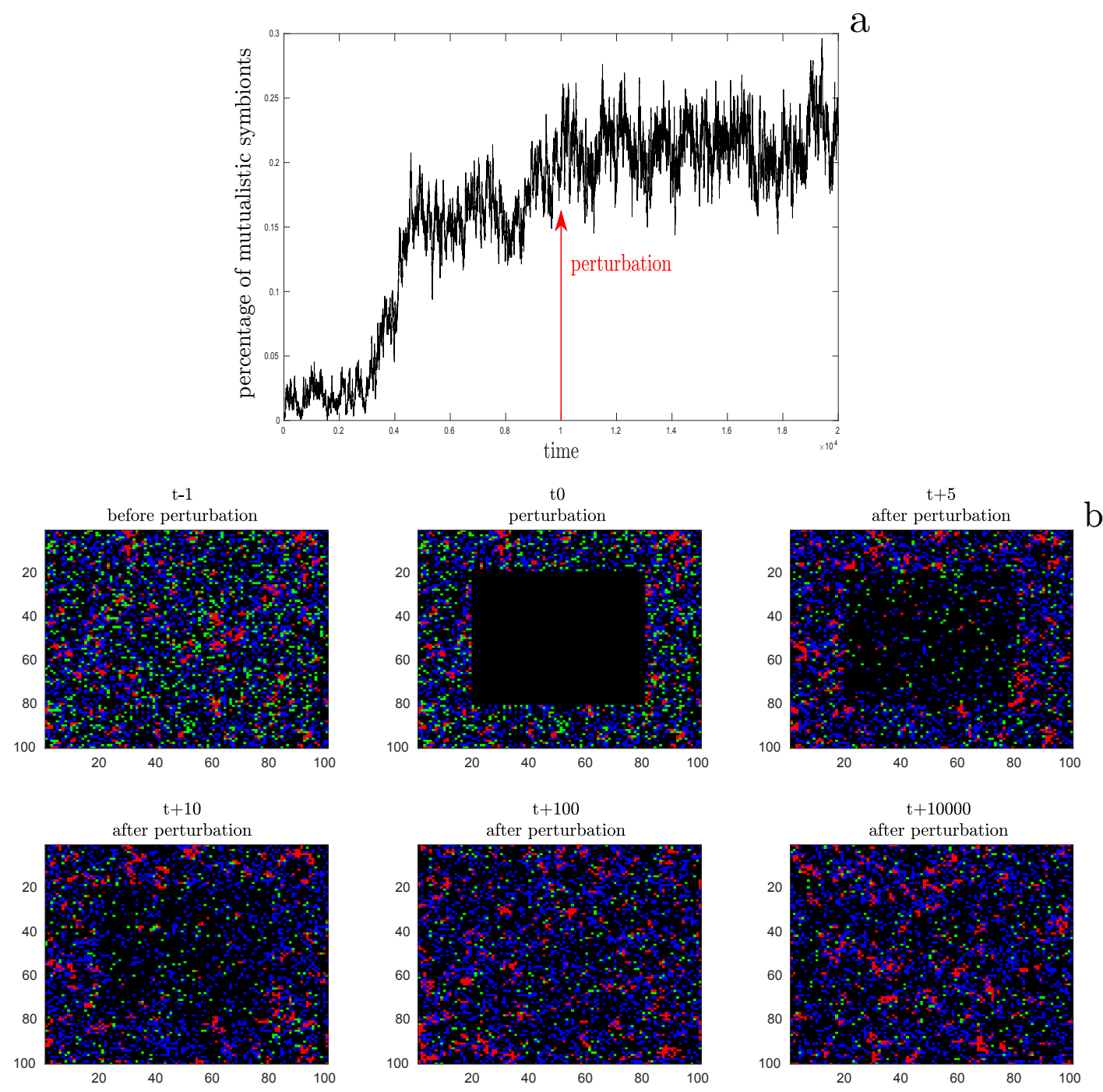

Figure A4: Maintenance of mutualism in the face of a reduction in competition caused by a perturbation creating a large square of free cells. a) The proportion of mutualist symbionts, showing from t0 to $t 10000$ the transition to mutualism and the eco-evolutionary equilibrium, then until $t 20000$ the response following the perturbation. b) Snapshot at several times: $t-1$ is the eco-evolutionary equilibrium with mutualism just before the perturbation, then at $t 0$ the perturbation, and then $t+5, t+10, t+100$ and $t+10000$ after the perturbation. In black, the free cells; in green, the hosts alone; in blue, the couples with parasitic symbionts; and in red, the couples with mutualist symbionts.

\section{A.3 Mortality and dispersal cost can induce host dependency in emerging mutualistic systems.}

In the main text, we focused on the effect of mortality and dispersal cost on the transition to mutualism and host dependency. Here, we present in more detail the effect of dispersal cost on the distribution of hosts and symbionts in trait space for three values of dispersal cost and fixed mortality rate (Figure A5). In addition, the table A2 shows the features of the clusters in the trait distribution.

We demonstrated that the dispersal cost favours the transition to mutualism. Moreover, even when the cost was high, the features of the clusters revealed that parasitic symbionts maintained a more global dispersal than mutualistic symbionts. 

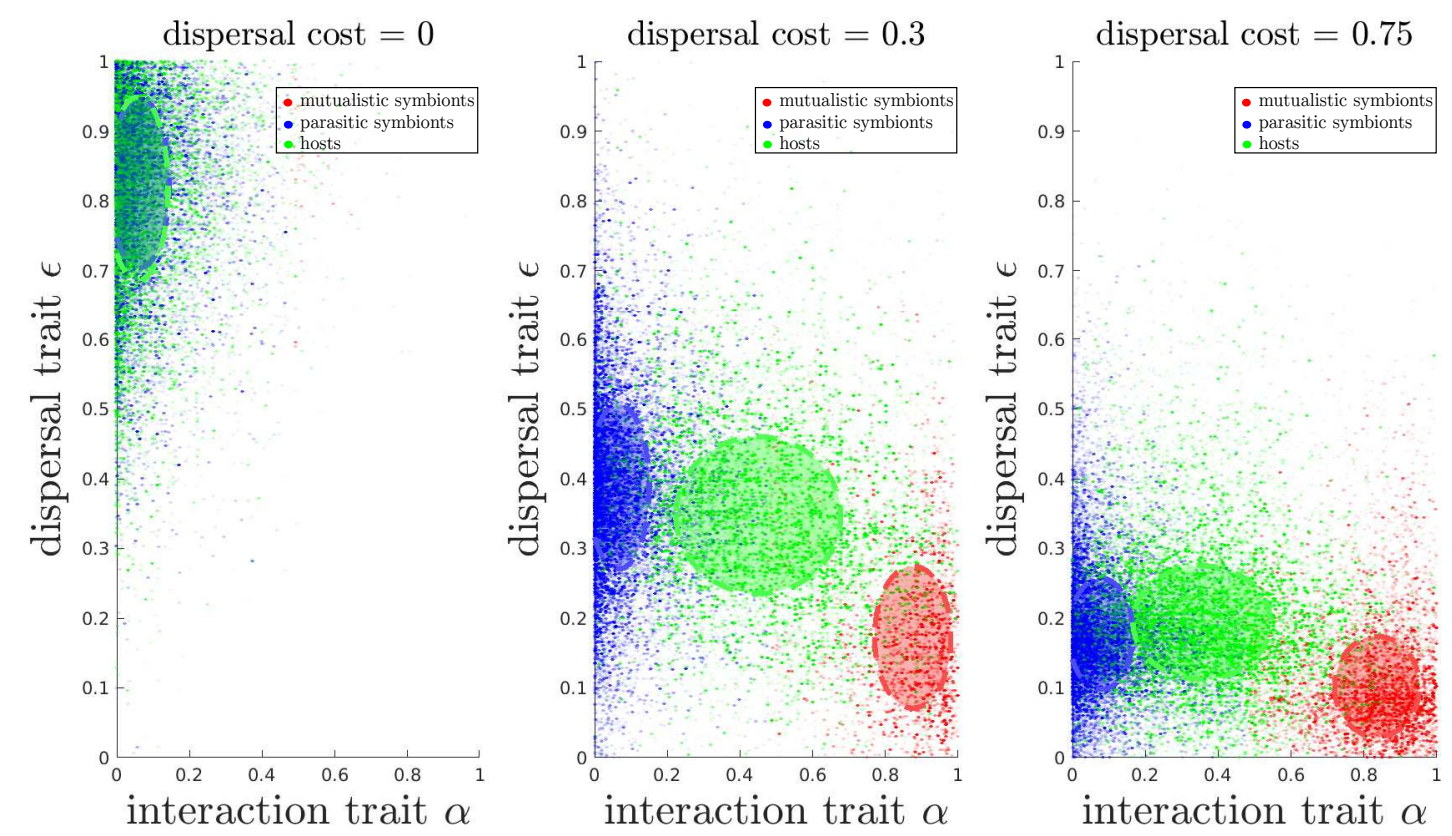

Figure A5: Joint distribution of the host (green), the parasitic symbiont (blue) and the mutualistic symbiont (red) populations in the traits domain for mortality $m=0.03$ and an increasing dispersal cost $d \in\{0,0.3,0.75\}$. The ellipses correspond to the standard deviation. The 48 runs averaged in Figure 6 are plotted together.

\begin{tabular}{cccc}
\hline dispersal cost & species & $\begin{array}{c}\text { mean } \\
\text { interaction trait }\end{array}$ & $\begin{array}{c}\text { mean } \\
\text { dispersal trait }\end{array}$ \\
\hline$d=0$ & hosts & 0.074 & 0.81 \\
& parasitic symbionts & 0.06 & 0.82 \\
& mutualistic symbionts & $*($ density $<2 \%)$ & $*($ density $<2 \%)$ \\
\hline$d=0.3$ & hosts & 0.45 & 0.35 \\
& parasitic symbionts & 0.07 & 0.39 \\
& mutualistic symbionts & 0.88 & 0.17 \\
\hline \multirow{2}{*}{$d=0.75$} & hosts & 0.36 & 0.19 \\
& parasitic symbionts & 0.08 & 0.18 \\
& mutualistic symbionts & 0.84 & 0.10 \\
\hline
\end{tabular}

Table A2: Features of the clusters in the traits domain 


\section{References}

E. Akçay. Evolutionary models of mutualism. In J.L. Bronstein, editor, Mutualism, pages 57-76. Oxford University Press, July 2015.

A. Akdeniz and M. van Veelen. The cancellation effect at the group level. Evolution, 74(7):1246-1254, 2020.

E. Akman Gündüz and A.E. Douglas. Symbiotic bacteria enable insect to use a nutritionally inadequate diet. Proceedings of the Royal Society B: Biological Sciences, 276(1658):987-991, 2009.

Erol Akçay. Population structure reduces benefits from partner choice in mutualistic symbiosis. Proceedings of the Royal Society B: Biological Sciences, 284(1850):2016-2317, 2017.

S. Alizon and P. Taylor. Empty sites can promote altruistic behavior. Evolution: International Journal of Organic Evolution, 62(6):1335-1344, 2008.

M.F. Allen. The ecology of mycorrhizae. Cambridge University Press, 1991.

R. Axelrod. The emergence of cooperation among egoists. The American Political Science Review, pages 306-318, 1981.

M. van Baalen and D.A. Rand. The unit of selection in viscous populations and the evolution of altruism. Journal of theoretical biology, 193(4):631-648, 1998.

G.M. Bennett and N.A. Moran. Heritable symbiosis: the advantages and perils of an evolutionary rabbit hole. Proceedings of the National Academy of Sciences, 112(33):10169-10176, 2015.

J.D. Bever, S.C. Richardson, B.M. Lawrence, J. Holmes, and M. Watson. Preferential allocation to beneficial symbiont with spatial structure maintains mycorrhizal mutualism. Ecology letters, 12(1):13-21, 2009.

M. Boots and M. Mealor. Local interactions select for lower pathogen infectivity. Science, 315(5816):1284-1286, 2007.

R.M. Borges. How to be a fig wasp parasite on the fig-fig wasp mutualism. Current Opinion in Insect Science, 8:34-40, 2015.

J.L. Bronstein. Conditional outcomes in mutualistic interactions. Trends in ecology $\mathcal{E}$ evolution, 9(6):214-217, 1994.

J.L. Bronstein. The contribution of ant-plant protection studies to our understanding of mutualism 1. Biotropica, 30(2):150-161, 1998.

J.L. Bronstein. Mutualism. Oxford University Press, USA, 2015.

J.L. Bronstein, R. Alarcón, and M. Geber. The evolution of plant-insect mutualisms. New Phytologist, 172(3): 412-428, 2006.

R.M. Callaway, R.W. Brooker, P. Choler, Z. Kikvidze, C.J. Lortie, R. Michalet, L. Paolini, F.I. Pugnaire, B. Newingham, E.T. Aschehoug, and C. Armas. Positive interactions among alpine plants increase with stress. Nature, 417(6891):844-848, 2002.

F. Débarre, S. Lion, M. Van Baalen, and S. Gandon. Evolution of host life-history traits in a spatially structured host-parasite system. The American Naturalist, 179(1):52-63, 2012.

R.F. Denison and E.T. Kiers. Why are most rhizobia beneficial to their plant hosts, rather than parasitic? Microbes and Infection, 6(13):1235-1239, 2004.

L. Després and N. Jaeger. Evolution of oviposition strategies and speciation in the globeflower flies chiastocheta spp.(anthomyiidae). Journal of Evolutionary Biology, 12(4):822-831, 1999.

G.C. Drew, E.J. Stevens, and K.C. King. Microbial evolution and transitions along the parasite-mutualist continuum. Nature Reviews Microbiology, pages 1-16, 2021.

D.P. Edwards, M. Hassall, W.J. Sutherland, and D.W. Yu. Selection for protection in an ant-plant mutualism: host sanctions, host modularity, and the principal-agent game. Proceedings of the Royal Society B: Biological Sciences, 273(1586):595-602, 2006.

S. Estrela, B. Kerr, and J.J. Morris. Transitions in individuality through symbiosis. Current opinion in microbiology, 31:191-198, 2016.

D. Feeny, F. Berkes, B.J. McCay, and J.M. Acheson. The tragedy of the commons: twenty-two years later. Human ecology, 18(1):1-19, 1990.

J-B. Ferdy and B. Godelle. Diversification of transmission modes and the evolution of mutualism. The American Naturalist, 166(5):613-627, 2005.

R.M. Fisher, C.K. Cornwallis, and S.A. West. Group formation, relatedness, and the evolution of multicellularity. Current Biology, 23(12):1120-1125, 2013.

K.R. Foster and T. Wenseleers. A general model for the evolution of mutualisms. Journal of evolutionary biology, 19(4):1283-1293, 2006.

H. Garrett. The tragedy of the commons. Science, 162(3859):1243-1248, 1968. 
M. Genkai-Kato and N. Yamamura. Evolution of mutualistic symbiosis without vertical transmission. Theoretical population biology, 55(3):309-323, 1999.

W.D. Hamilton. The genetical evolution of social behaviour. ii. Journal of theoretical biology, 7(1):17-52, 1964a. W.D. Hamilton. The genetical evolution of social behaviour. i. Journal of Theoretical Biology, 7(1):1-16, 1964b.

W. Harcombe. Novel cooperation experimentally evolved between species. Evolution: International Journal of Organic Evolution, 64(7):2166-2172, 2010.

G. Hardin. Extensions of "the tragedy of the commons". Science, 280(5364):682-683, 1998.

E.A. Herre, N. Knowlton, U.G. Mueller, and S.A. Rehner. The evolution of mutualisms: exploring the paths between conflict and cooperation. Trends in ecology \& evolution, 14(2):49-53, 1999.

M.E. Hochberg, D.J. Rankin, and M. Taborsky. The coevolution of cooperation and dispersal in social groups and its implications for the emergence of multicellularity. BMC Evolutionary Biology, 8(1):1-14, 2008.

N.C. Johnson, J-H. Graham, and F.A. Smith. Functioning of mycorrhizal associations along the mutualismparasitism continuum. New phytologist, 135(4):575-585, 1997.

S. Kéfi, M. Rietkerk, M. van Baalen, and M. Loreau. Local facilitation, bistability and transitions in arid ecosystems. Theoretical population biology, 71(3):367-379, 2007.

S. Kéfi, M. van Baalen, M. Rietkerk, and M. Loreau. Evolution of local facilitation in arid ecosystems. The American Naturalist, 172(1):E1-E17, 2008.

B. Kerr and J. Nahum. The evolution of restraint in structured populations: setting the stage for an egalitarian major transition. In The major transitions in evolution revisited, pages 127-140. MIT Press, Cambridge, 2011.

B. Kerr, C. Neuhauser, Brendan J.M. Bohannan, and A.M. Dean. Local migration promotes competitive restraint in a host-pathogen'tragedy of the commons'. Nature, 442(7098):75-78, 2006.

E.T. Kiers, R.A. Rousseau, S.A. West, and R.F. Denison. Host sanctions and the legume-rhizobium mutualism. Nature, 425(6953):78-81, 2003.

K.C. King, M.A. Brockhurst, O. Vasieva, S. Paterson, A. Betts, S.A. Ford, C.L. Frost, M.J. Horsburgh, S. Haldenby, and G.DD. Hurst. Rapid evolution of microbe-mediated protection against pathogens in a worm host. The ISME journal, 10(8):1915-1924, 2016.

J.C. Koella. The spatial spread of altruism versus the evolutionary response of egoists. Proceedings of the Royal Society of London. Series B: Biological Sciences, 267(1456):1979-1985, 2000.

K. Laland, B. Matthews, and M.W. Feldman. An introduction to niche construction theory. Evolutionary ecology, 30(2):191-202, 2016.

J.F Le Galliard, R. Ferriere, and U. Dieckmann. Adaptive evolution of social traits: origin, trajectories, and correlations of altruism and mobility. The American Naturalist, 165(2):206-224, 2005.

R.C. Lewontin. Organism and environment. ic plotkin (ed.), learning, development and culture: Essays in evolutionary epistemology, 1982.

R.C. Lewontin. Gene, organism and environment. Evolution from molecules to men, 273:285, 1983.

S. Lion and M. van Baalen. From infanticide to parental care: why spatial structure can help adults be good parents. The American Naturalist, 170(2):E26-E46, 2007.

S. Lion and M. van Baalen. Self-structuring in spatial evolutionary ecology. Ecology letters, 11(3):277-295, 2008.

K. ML. Mack. Selective feedback between dispersal distance and the stability of mutualism. Oikos, 121(3): 442-448, 2012.

F.T. Maestre, S. Bautista, and J. Cortina. Positive, negative, and net effects in grass-shrub interactions in mediterranean semiarid grasslands. Ecology, 84(12):3186-3197, 2003.

S. Maynard. Evolutionary genetics. Oxford University Press, 1998. ISBN 9780198502319.

M.J. McFall-Ngai. The importance of microbes in animal development: lessons from the squid-vibrio symbiosis. Annual review of microbiology, 68:177-194, 2014.

J. Mitteldorf and D.S. Wilson. Population viscosity and the evolution of altruism. Journal of theoretical biology, 204(4):481-496, 2000.

C. Mullon, L. Keller, and L. Lehmann. Social polymorphism is favoured by the co-evolution of dispersal with social behaviour. Nature ecology $\&$ evolution, 2(1):132-140, 2018.

F John Odling-Smee, Kevin N Laland, and Marcus W Feldman. Niche construction: the neglected process in evolution (MPB-37). Princeton university press, 2013.

J.W. Pepper. Simple models of assortment through environmental feedback. Artificial life, 13(1):1-9, 2007.

J.W. Pepper and B.B. Smuts. A mechanism for the evolution of altruism among nonkin: positive assortment through environmental feedback. The American Naturalist, 160(2):205-213, 2002.

J. Purcell, A. Brelsford, and L. Avilés. Co-evolution between sociality and dispersal: the role of synergistic 
cooperative benefits. Journal of Theoretical Biology, 312:44-54, 2012.

D.C. Queller. Cooperators since life began. The Quarterly Review of Biology, 72(2), 1997.

D.C. Queller. Relatedness and the fraternal major transitions. Philosophical Transactions of the Royal Society of London. Series B: Biological Sciences, 355(1403):1647-1655, 2000.

D.C. Queller and J.E. Strassmann. Problems of multi-species organisms: endosymbionts to holobionts. Biology ES Philosophy, 31(6):855-873, 2016.

V. Rico-Gray and P.S. Oliveira. The ecology and evolution of ant-plant interactions. University of Chicago Press, 2008.

J.L. Sachs and E.L. Simms. Pathways to mutualism breakdown. Trends in ecology 85 evolution, 21(10):585-592, 2006.

J.L. Sachs, U.G. Mueller, T.P. Wilcox, and J.J. Bull. The evolution of cooperation. The Quarterly review of biology, 79(2):135-160, 2004.

J.L. Sachs, R.G. Skophammer, and J.U. Regus. Evolutionary transitions in bacterial symbiosis. Proceedings of the National Academy of Sciences, 108(Supplement 2):10800-10807, 2011.

J.W. Shapiro and P.E. Turner. Evolution of mutualism from parasitism in experimental virus populations. Evolution, 72(3):707-712, 2018.

J.W. Shapiro, E.SCP. Williams, and P.E. Turner. Evolution of parasitism and mutualism between filamentous phage m13 and escherichia coli. PeerJ, 4:e2060, 2016.

E. Szathmáry. Toward major evolutionary transitions theory 2.0. Proceedings of the National Academy of Sciences, 112(33):10104-10111, 2015.

E. Szathmáry and John M. Smith. The major transitions in evolution. WH Freeman Spektrum Oxford, UK:, 1995.

P.D. Taylor and A.J. Irwin. Overlapping generations can promote altruistic behavior. Evolution, 54(4):1135-1141, 2000.

J.N. Thompson and B.M. Cunningham. Geographic structure and dynamics of coevolutionary selection. Nature, 417(6890):735-738, 2002.

J.M.J. Travis, R.W. Brooker, E.J. Clark, and C. Dytham. The distribution of positive and negative species interactions across environmental gradients on a dual-lattice model. Journal of Theoretical Biology, 241(4): 896-902, 2006.

B. Week and S.L. Nuismer. Coevolutionary arms races and the conditions for the maintenance of mutualism. The American Naturalist, 198(2):000-000, 2021.

A.R. Weeks, M. Turelli, W.R. Harcombe, K.T. Reynolds, and A.A. Hoffmann. From parasite to mutualist: rapid evolution of wolbachia in natural populations of drosophila. PLoS Biol, 5(5):e114, 2007.

G.DA. Werner, W.K. Cornwell, J.HC. Cornelissen, and E.T. Kiers. Evolutionary signals of symbiotic persistence in the legume-rhizobia mutualism. Proceedings of the National Academy of Sciences, 112(33):10262-10269, 2015.

G.D.A. Werner, J.H.C. Cornelissen, W.K. Cornwell, N.A. Soudzilovskaia, J. Kattge, S.A. West, and E.T. Kiers. Symbiont switching and alternative resource acquisition strategies drive mutualism breakdown. Proceedings of the National Academy of Sciences, 115(20):5229-5234, 2018.

S.A. West, E.T. Kiers, E.L. Simms, and R.F. Denison. Sanctions and mutualism stability: why do rhizobia fix nitrogen? Proceedings of the Royal Society of London. Series B: Biological Sciences, 269(1492):685-694, 2002.

D.M. Wilkinson. The role of seed dispersal in the evolution of mycorrhizae. Oikos, pages 394-396, 1997.

D.M. Wilkinson and T.N. Sherratt. Horizontally acquired mutualisms, an unsolved problem in ecology? Oikos, 92(2):377-384, 2001.

D.S. Wilson and L.A. Dugatkin. Group selection and assortative interactions. The American Naturalist, 149(2): 336-351, 1997.

D.S. Wilson and E. Sober. Reintroducing group selection to the human behavioral sciences. Behavioral and brain sciences, 17(4):585-607, 1994.

D.S. Wilson, G.B. Pollock, and L.A. Dugatkin. Can altruism evolve in purely viscous populations? Evolutionary ecology, 6(4):331-341, 1992.

N. Yamamura, M. Higashi, N. Behera, and J.Y. Wakano. Evolution of mutualism through spatial effects. Journal of theoretical biology, 226(4):421-428, 2004. 